J. Urol. Urogynäkol. AT 2020 · 27:15-17 https://doi.org/10.1007/s41972-020-00099-2 Online publiziert: 24. März 2020

(c) Der/die Autor(en) 2020

\section{Heinz Kölbl · Ksenia Halpern}

Abteilung für Allgemeine Gynäkologie und Gynäkologische Onkologie, Universitätsklinik für Frauenheilkunde, Allgemeines Krankenhaus, Wien, Österreich

\title{
Bandoperation bei betagten Frauen - Nutzen/Risiko?
}

\section{Harninkontinenz - Grundlagen}

An der globalen demografischen Entwicklung ist die Harninkontinenz im Alter als ein deutlich zunehmendes medizinisches und sozioökonomisches Problem zu erkennen, welches auch in Ländern der sog. westlichen Welt häufiger vorkommt als gedacht. Von den in Deutschland lebenden Menschen mit einer behandlungswürdigen Harninkontinenz beispielsweise sind mehr als 2 Mio. älter als 60 Jahre $(11 \%$ dieser Altersgruppe), bei den über 80-Jährigen sind es nahezu $30 \%$ [1]. Ursachen sind u. a. fibromuskuläre Veränderungen, Störungen der neurogenen Steuerung von Harnblase und Sphinktermuskulatur sowie altersbedingte Veränderungen der Anatomie des unteren Harntrakts. Als weitere Faktoren kommen medikamentöse Nebenwirkungen in Frage - meist aufgrund einer Multimedikation wegen Komorbiditäten in dieser Generation [2]. Auch findet sich im Alter häufiger eine Kombination von Stressund Dranginkontinenz (sog. Mischinkontinenz).

Für die Feststellung einer Harninkontinenz braucht es in der Regel nicht mehr als eine Basisdiagnostik. Zu dieser gehören neben gezielter Anamnese und klinischer Untersuchung (inklusive klinischem Stresstest) ein Harnbefund, die Restharnbestimmung und ein Miktionsprotokoll. Im Zuge des ärztlichen $\mathrm{Ge}$ sprächs ist es auch unverzichtbar, die Ziele und Wünsche der Patientinnen zu erfahren. Vielen von ihnen genügt es nämlich durchaus, eine sog. soziale Kontinenz zu erreichen, welche ihnen ein gut integriertes Leben in ihrem gesellschaftlichen Umfeld erlaubt.
Neben der medikamentösen Therapie der Harninkontinenz kommt physikalischen Maßnahmen und Verhaltensinterventionen eine große Bedeutung zu. In einer Cochrane-Analyse aus 13 randomisierten Studien mit insgesamt 714 Frauen wurde der Effekt der Beckenbodengymnastik gegenüber Plazebo bei weiblicher Belastungsinkontinenz untersucht. Die Wahrscheinlichkeit, wieder kontinent $\mathrm{zu}$ werden, war in der Verumgruppe um das 17-Fache und somit signifikant erhöht [3].

Erweist sich die konservative Therapie jedoch als zu wenig wirksam, ist auch bei betagten Patientinnen eine operative Intervention durchaus zu erwägen, sofern aus internistischer Sicht grundsätzlich eine Operationsfähigkeit gegeben ist. Indikation für die Implantation eines TVT z. B. sind die Belastungsinkontinenz oder eine Mischinkontinenz mit hohem Belastungsanteil.

\section{Chirurgie mit Bändern oder Netzen}

Als elegante und kaum belastende Operation zur Korrektur der weiblichen Stressharninkontinenz wurde die Methode des „tension-free vaginal tape“ (TVT) erstmals 1996 von Ulf Ulmsten et al. beschrieben [4]. Seit 1998 wird sie auch in Österreich eingesetzt und bewährte sich als höchst effektive Therapie.

Nach den positiven Erfahrungen mit dem TVT in der Chirurgie der Inkontinenz wurden alloplastische Materialien in Form von Netzen oder "meshes“ in den letzten Jahren auch für andere rekonstruktive Eingriffe am Beckenboden eingesetzt. Allerdings verhalten sich die verschiedenen Typen von Netzen (synthetisch, nichtresorbierbar) auch im Gewebe unterschiedlich. Entsprechend der Meshklassifikation nach Amid aus dem Jahr 1997 [5] wissen wir heute, dass anstelle von makroporösen Netzen vom Typ 1 (Porengröße $>75 \mu$ ) anderen Netztypen, insbesondere mikroporösen und/ oder multifilamentären Materialien, der Vorzug zu geben ist. Neuerdings werden auch biologische Netzmaterialien eingesetzt, deren Wert sich im klinischen Alltag noch zu beweisen haben wird.

In der Therapie der Belastungsinkontinenz ist die Einlage von Polypropylenbändern unter die mittlere Harnröhre gegenwärtig die häufigste Operation. Es sind zahlreiche Produkte auf dem Markt, die sich hinsichtlich des Materials unterscheiden. Hinzu kommt, dass auch verschiedene Zugangswege und Operationstechniken angegeben werden. Ein Beispiel dafür ist das TVT-O (transobturatorisches TVT).

Für die klassische TVT-Operation liegen jedenfalls bereits Langzeitdaten über 10 Jahre vor sowie ein Komplikationsregister und Kosten-Nutzen-Analysen. $\mathrm{Na}$ turgemäß existieren für die früher meist geübte Kolposuspension heute mehr Daten, welche über das Langzeit-Follow-up und die Rate der Rezidive informieren. Aus dem Vergleich der Zahlen lässt sich verlässlich schließen, dass das TVT mit einer Langzeitheilungsrate von $81 \%$ genauso effektiv ist.

\section{TVT bei älteren Patientinnen}

Angesichts der Prävalenz der Belastungsinkontinenz im demografischen Segment der älteren Frau einerseits und der routinemäßigen Anwendung des TVT andererseits, erscheint es uns von prakti- 
schem Interesse, Besonderheiten in dieser Konstellation herauszufinden. Zu diesem Zweck führten wir eine selektive Recherche der wissenschaftlichen Literatur in PubMed durch - dies mit den Stichworten „urinary incontinence“, „tension free vaginal tape“ und „stress incontinence AND elderly“.

Unter der selbstverständlichen Voraussetzung einer korrekten Indikation hängt die Entscheidung zur Implantation eines TVT bei älteren Patientinnen von spezifischen Faktoren ab: Der Wunsch nach dem Eingriff (welchen jüngere Frauen naturgemäß ohne langes Überlegen artikulieren) muss klar geäußert werden. Ferner sind Komorbiditäten zu berücksichtigen, d.h. dass insbesondere auch die allgemeine Operabilität sorgfältigst zu prüfen ist.

Aus den Studien geht hervor, dass das Verfahren unter den vorgenannten Bedingungen auch bei älteren Patientinnen sicher und sehr effektiv ist. Die Langzeitergebnisse der TVT-Methode zeigen über 11 Jahre eine persistierende Kontinenzrate von mehr als $80 \%$ [6, 7]. Auffallend ist eine erhöhte Rate von postoperativen Harnwegsinfekten und De-novoDranginkontinenz [8], während schwerere Komplikationen eher selten sind: An erster Stelle zu nennen sind hier die Blasenperforation bei TVT und chronische Schmerzen im Oberschenkel nach TVT-O [8].

Kontraindikationen für die TVTOperation bei älteren Patienten sind wohl all jene, bei denen sich auch andere Eingriffe verbieten würden, wie schwere kardiale Erkrankungen, Blutgerinnungsstörungen usw. Allerdings können Ältere auch davon profitieren, dass das TVT unter Lokal- bzw. Spinalanästhesie eingesetzt werden kann.

\section{Schlussfolgerung}

Bei Versagen der konservativen Therapie ist das TVT mit $80 \%$ iger Erfolgsrate auch in der Langzeitbeobachtung eine sichere und effektive chirurgische Option bei Stressharninkontinenz von betagten Patientinnen. In der Indikationsstellung zu berücksichtigen sind natürlich die in dieser Generation häufigen Komorbiditäten. Auch sind die Patientinnen über
J. Urol. Urogynäkol. AT 2020 · 27:15-17 https://doi.org/10.1007/s41972-020-00099-2

(c) Der/die Autor(en) 2020

\section{H. Kölbl·K. Halpern}

\section{Bandoperation bei betagten Frauen - Nutzen/Risiko?}

\section{Zusammenfassung}

Die Implantation spannungsfreier Bänder ("tension free vaginal tape" [TVT]) ist eine minimalinvasive Technik zur Behandlung der Belastungsinkontinenz. Um den Stellenwert und die Besonderheiten der Methode bei älteren Menschen zu evaluieren, wurde eine selektive Literaturrecherche in PubMed mit den Stichworten „urinary incontinence $/$ /"tension free vaginal tape $/$ ",stress incontinence" AND „elderly“ durchgeführt. Angesichts der demografischen Entwicklung weltweit erweist sich die Harninkontinenz im Alter als zunehmendes medizinisches und sozioökonomisches Problem. Ein TVT kann selbst bei Hochaltrigen unter folgenden Bedingungen ins Auge gefasst werden: grundsätzlich fehlende Kontraindikation gegen einen chirurgischen Eingriff; Vorliegen einer Belastungs- oder Mischinkontinenz (mit hohem Belastungsanteil); Versagen der konservativen Therapie. Unter der Voraussetzung von Operationswunsch und gegebener Operabilität zeigen die Langzeitergebnisse des TVT über 11 Jahre eine persistierende Kontinenzrate von mehr als $80 \%$. Die Operation ist sicher und effektiv, geht allerdings mit einer erhöhten Rate von postoperativen Harnwegsinfekten und DeNovo-Dranginkontinenz einher.

\section{Schlüsselwörter}

Spannungsfreie Bänder · „Tension-free vaginal tape" (TVT) · Belastungsinkontinenz · Ältere Patientinnen

\section{The tension-free vaginal tape procedure in elderly women: what is the benefit/risk?}

\section{Abstract}

The implantation of tension-free vaginal tape (TVT) is a minimally invasive technique for the treatment of stress urinary incontinence. In order to evaluate particular features of the method in the elderly, a literature search was performed in PubMed with the keywords "urinary incontinence"/"tension free vaginal tape"/"stress incontinence" AND "elderly". In view of demographic developments worldwide, urinary incontinence is emerging as an increasing medical and socio-economic problem. The possibility of TVT can be considered even in very elderly individuals under the following conditions: no genera contraindications to surgery; presence of stress or mixed incontinence (with a strong stress component); failure of conservative therapy. Under the prerequisite of a clear desire for surgery and assuming operability, the long-term results of TVT show a lasting rate of continence of over $80 \%$ at 11 years. The operation is safe and effective; however, it is associated with a higher incidence of postoperative urinary infections and de-novo urge incontinence.

\section{Keywords}

Tension-free tapes - Tension-free vaginal tape (TVT) - Stress urinary incontinence - Older patients das postoperative Risiko von häufigeren Harnwegsinfekten und De-novo-Dranginkontinenz aufzuklären. Um schwerere Komplikationen gerade bei älteren Patientinnen so gut wie möglich hintanzuhalten, sollte der Eingriff in diesen Fällen Operateuren mit hoher Expertise vorbehalten sein.

\section{Fazit für die Praxis}

- Das TVT („tension-free vaginal
tape“) ist für die Behandlung der
Stressinkontinenz auch für ältere
Patientinnen durchaus geeignet.
- Die Langzeitergebnisse von $80 \%$
Kontinenzrate sind ermutigend.
- Die in dieser Generation häufigen
Komorbiditäten sind in der Indikati-
onsstellung zu berücksichtigen.




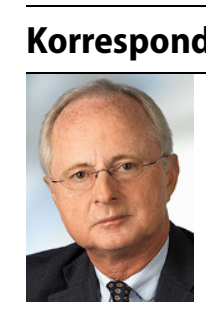

Univ. Prof. Dr.

Abteilung für Allgemeine

Gynäkologie und

Gynäkologische Onkologie,

Universitätsklinik für

Frauenheilkunde,

Allgemeines Krankenhaus

Währinger Gürtel 18-20,

1090 Wien, Österreich

heinz.koelbl@

meduniwien.ac.at

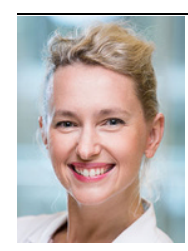

Dr. Ksenia Halpern

Abteilung für Allgemeine

Gynäkologie und

Gynäkologische Onkologie,

Universitätsklinik für

Frauenheilkunde,

Allgemeines Krankenhaus

Währinger Gürtel 18-20,

1090 Wien, Österreich

ksenia.halpern@

meduniwien.ac.at

Funding. Open access funding provided by Medical University of Vienna.

\section{Einhaltung ethischer Richtlinien}

Interessenkonflikt. H. Kölbl und K. Halpern geben an, dass kein Interessenkonflikt besteht.

Für diesen Beitrag wurden von den Autoren keine Studien an Menschen oder Tieren durchgeführt. Für die aufgeführten Studien gelten die jeweils dort angegebenen ethischen Richtlinien.

Open Access Dieser Artikel wird unter der Creative Commons Namensnennung 4.0 International Lizenz veröffentlicht, welche die Nutzung, Vervielfältigung, Bearbeitung, Verbreitung und Wiedergabe in jeglichem Medium und Format erlaubt, sofern Sie den/die ursprünglichen Autor(en) und die Quelle ordnungsgemäß nennen, einen Link zur Creative Commons Lizenz beifügen und angeben, ob Änderungen vorgenommen wurden.

Die in diesem Artikel enthaltenen Bilder und sonstige Drittmaterial unterliegen ebenfalls der genannten Creative Commons Lizenz, sofern sich aus der Abbildungslegende nichts anderes ergibt. Sofern das betreffende Material nicht unter der genannten Creative Commons Lizenz steht und die betreffende Handlung nicht nach gesetzlichen Vorschriften erlaubt ist, ist für die oben aufgeführten Weiterverwendungen des $\mathrm{Ma}$ terials die Einwilligung des jeweiligen Rechteinhabers einzuholen.

Weitere Details zur Lizenz entnehmen Sie bitte de Lizenzinformation auf http://creativecommons.org/ licenses/by/4.0/deed.de.

\section{Literatur}

1. Weltz-Barth A (2007) Inkontinenz im Alter, ein soziales und ökonomisches Problem. Urologe 46:363-364

2. Talasz H, Wunderlich M (2020) Multimedikation und Kontinenz beim geriatrischen Patienten. JUro Urogynäkol AT. https://doi.org/10.1007/s41972020-00096-5

3. Dumoulin C, Hay-Smith J (2008) Pelvic floor muscle training versus no treatment for urinary incontinence in women. A Cochrane systematic review. Eur J Phys Rehabil Med 44:47

4. Ulmsten U, Henriksson L, Johnson P, Varhos G (1996) An ambulatory surgical procedure under local anesthesia for treatment of female urinary incontinence. Int Urogynecol J Pelvic Floor Dysfunct 7:81-85

5. Amid PK (1997) Classification of biomaterials and their related complications in abdominal wall hernia surgery. Hernia 1:15-21. https://doi.org/10. 1007/BF02426382

6. Nilsson CG, Palva K, Rezapour M et al (2008) Eleven years prospective follow-up of the tension free vaginal tape procedure for treatment of stress urinary incontinence. Int Urogynecol J Pelvic Floor Dysfunct 19:1043

7. Tamussino K, Hanzal E, Kölle D et al. (2001) The Austrian Tension-Free Vaginal Tape Registry. Int Urogynecol J 12:28-30. https://doi.org/10.1007/ s001920170009

8. Groutz A, Cohen A, Gold R, Pauzner D, Lessing JB, Gordon D (2011) The safety and efficacy of the "inside-out" trans-obturator TVT in elderly versus younger stress-incontinent women: a prospective study of 353 consecutive patients. Neurourol Urodyn 30(3):380-383

Hinweis des Verlags. Der Verlag bleibt in Hinblick auf geografische Zuordnungen und Gebietsbezeichnungen in veröffentlichten Karten und Institutsadressen neutral.

\section{Perioperative Medizin}

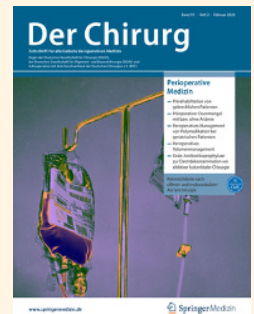

Wie jeder chirurgisch tätige Arzt weiß, ist mit eine technisch gelungenen Operation der Behandlungs erfolg lange nicht sichergestellt. Neben der richtigen

Indikationsstellung müssen chirurgische Patienten somatisch und psychisch auf eine Operation vorbereitet und nach der Operation adäquat rehabilitiert werden. In Der Chirurg 02/2020 erhalten Sie einen Überblick über aktuelle Aspekte der perioperativen Medizin in der Allgemeinund Viszeralchirurgie, der sie bei der täglichen Arbeit mit ihren Patienten hilfreich unterstützen kann.

- Prärehabilitation von gebrechlichen Patienten

- Präoperativer Eisenmangel mit bzw.ohne Anämie

- Polymedikation bei geriatrischen Patienten: Abstimmung mit dem Hausarzt

- Perioperatives Volumenmanagement

- Orale Antibiotikaprophylaxe zur Darmdekontamination vor elektiver kolorektaler Chirurgie

Suchen Sie noch mehr zum Thema? Mit e.Med - den maßgeschneiderten Fortbildungsabos von Springer Medizin - haben Sie Zugriff auf alle Inhalte von SpringerMedizin.de. Sie können schnell und komfortabel in den für Sie relevanten Zeitschriften recherchieren und auf alle Inhalte im Volltext zugreifen.

Weitere Infos zu e.Med finden Sie auf springermedizin.de unter "Abos" 